\title{
Comparative Analysis of Coverage Schemes in WSN
}

\author{
Vedang Ratan Vatsa \\ Maharshi Dayanand University \\ Rohtak, India
}

\author{
Ankit Punia \\ Maharshi Dayanand University \\ Rohtak, India
}

\author{
Shruti Vatsa \\ Kurukshetra University \\ Kurukshetra, India
}

\begin{abstract}
Wireless sensor systems (WSN), one of the prevailing innovation patterns, are presently increasing noteworthy consideration from the analysts attributable to its extensive variety of utilizations identified with horticulture, observation, human services and natural checking. One of the difficulties of the remote sensor systems is that of scope while keeping availability. Scope is considered as a measure of nature of administration (QoS) in a sensor system as it decides how well every point in the district is checked and followed by sensors and how precise is the data accumulated by the hubs. In this paper, we characterize a few terms and ideas of scope issue and some essential outline contemplations in scope of WSN. We additionally give a brief rundown and examination of existing scope plans.
\end{abstract}

\section{Keywords}

WSN, Coverage Scheme, ASCENT, OGDC, LDAC

\section{INTRODUCTION}

A Wireless Sensor Network (Akyildiz, Su, Sankarasubramaniam, \& Cayirci, 2002) comprises of various remote self-sufficient sensor hubs. These hubs are highlighted by being small in size with restricted vitality or power more often than not supplied by a battery, low in expense and ready to perform numerous capacities. A WSN, spread over an expansive territory, comprises of a great many sensor hubs sent either haphazardly or deterministically over area of interest (ROI).

A sensor hub independent from anyone else has a few asset requirements, for example, restricted memory, battery force, sign preparing, calculation and correspondence capacities; subsequently it can sense just a little parcel of nature. Then again, a mix of these little sensors can accomplish a much greater assignment adequately and productively.

These hubs can sense and gather information from nature, perform some handling on assembled information and speak with one another remotely over short separations to perform accumulation and after that course the totaled information to base stations which can further connection the information to the outside world by means of the Internet or satellites.

An extensive variety of potential utilizations of WSN incorporate temperature and ecological conditions checking, untamed life living space observing, woods fire identification, security reconnaissance in military and front lines, social insurance, agribusiness and so on. Case in point (Polastre, Szewczyk, Mainwaring, Culler, \& Anderson, 2004), A system of sensor hubs can be introduced in some backwoods to control when a flame has begun.

The hubs will be furnished with sensors to control temperature, mugginess and gasses which are delivered by flame in the trees or vegetation. The early identification is critical for a fruitful activity of the firefighters; all made conceivable because of Wireless Sensor Networks, the fire detachment will have the capacity to know when a fire is begun and how it is spreading.

A standout amongst the most dynamic exploration fields in remote sensor systems is that of scope. Scope is normally based on the inquiry "how well the objective is checked or followed by sensors which together constitutes a sensor system?" It is a measure of Quality of administration (QoS) of detecting capacity for a sensor system.

The fundamental objective of this issue is to have every area in the focused on physical space inside of detecting scope of at least one sensor. Scope Problem (Chi-Fu \& Yu-Chee, 2005) in WSN, fundamentally is brought on by three principle reasons insufficient sensors to cover the entire locale of interest(ROI), restricted detecting extent and irregular arrangement of sensor hubs.

Notwithstanding scope, it is critical for a sensor system to look after network. Availability can be termed as the capacity of the sensor hubs to achieve the information sink or base station. On the off chance that there is no accessible course from a sensor hub to the information sink then the information gathered by that hub can't be prepared.

Both availability and scope issues (Zhou, Das, \& Gupta, 2004) are brought on by the restricted correspondence and detecting reach.

To tackle both issues, the arrangement lies in how the sensors are situated as for one another. It is a one of a kind issue, in amplifying scope the sensors should be set not very near one another so that the detecting capacity of the system is completely used furthermore, they must not be found too a long way from one another to stay away from the development of scope gaps (zone outside detecting scope of sensors).

Then again from network perspective, the sensors should be set sufficiently close so they are inside of one another's correspondence extent and therefore, availability is guaranteed. The real piece of this paper presents brief portrayal and correlation of the current scope plans for WSN. The point is to give a superior comprehension of scope innovation and its ideas.

The rest of this paper is sorted out as takes after. In next segment, some outline issues in scope issue are examined. Next, we arrange scope into three classifications: point scope, zone scope and way scope. After this, a synopsis and relative analysis of existing scope plans for WSN has been finished. At last, we talk about the conclusion. Toward the end of the paper is a rundown of references. 


\section{SCOPE}

Wireless ad hoc networks of battery controlled miniaturized scale sensors are multiplying quickly and changing the way data is assembled, prepared and imparted. These systems are imagined to have several reasonable sensors with detecting, information preparing and correspondence parts.

They normally work in unattended mode, convey over short separations and use multi jump correspondence. Numerous difficulties are acquainted due with the restricted vitality, extensive number of sensors, antagonistic workplace and nature of flighty arrangement of the sensor system.

Vitality preservation is observed to be the principal among them since it is regularly taken a toll restrictive or infeasible to renew the vitality of the sensors. This vitality issue in remote sensor systems stays one of the real obstructions some way or another keeping the complete abuse of this innovation. Effective Energy Management is ended up being the key prerequisite for the tenable configuration of a remote sensor system.

The general structure for vitality productive information procurement depends on an obligation cycle methodology requiring the sensor to be exchanged OFF amid unmoving time. Element Power Management is a compelling device in diminishing the framework power utilization without corrupting the execution.

The nature of remote channels or the system topology can change quickly even in a static system. Steering conventions ought to rapidly identify these flow and take measures to keep up hearty and effective directing ways. This should be considered in the configuration of a remote sensor system. In addition, a cross layer configuration on force mindful correspondence, considering the time changing nature of remote channels, is expected to upgrade the vitality use. In this work, all the aforementioned thoughts are fused. Here every sensor hub wisely gets to the remote medium and correspondence action is lessened for those sensor hubs, under poor channel conditions.

Thus the time differing nature of remote channels are considered in this work, in the enhancement of vitality administration in a remote sensor system.

- For an increment in the hub thickness, Energy Consumption expanded for AEMAC, SMAC and ZMAC plans, because of the increment in directing included. AEMAC plan showed the most minimal Energy Consumption, since more hubs are conveyed to the rest state, on an increment in the hub thickness. In addition, the compulsory wake up included in the other force sparing plans, is not utilized here prompting its better execution contrasted with alternate plans. ZMAC plan demonstrated the greatest Energy Consumption.

- For an increment in hub thickness, Throughput diminished for AEMAC, SMAC and ZMAC plans. This may be because of the all the more directing choices included on an increment in hub thickness bringing about less portion of the channel limit being utilized for information transmission. The enhanced technique of the AEMAC plan prompted its expanded throughput.

- On an increment in hub thickness, Delay diminishes for all the three plans AEMAC, SMAC and ZMAC. This is because of the vast number of hubs getting included in directing, which brings about faster information conveyance to the sink through these hubs. Later Delay settles to an enduring quality in all the three plans. AEMAC plan displayed the most reduced Delay and SMAC plan demonstrated the biggest Delay.

- An increment in Transmission Rate gave a reduction in Energy Consumption for all the three plans AEMAC, SMAC and ZMAC; since every one of the plans utilize their energy protection strategies viably in transmitting the vast number of information parcels transmitted per unit of time. In addition, the start up vitality overhead additionally diminishes on an increment in the Transmission Rate. AEMAC plan demonstrated the most reduced Energy Consumption and ZMAC plan demonstrated the most noteworthy Energy Consumption. So an increment in Transmission Rate can be selected as a decent technique to diminish the Energy Consumption in a remote sensor system.

- On an increment in the Transmission Rate, Throughput increments for AEMAC, SMAC and ZMAC plans on the grounds that the huge measure of information which is transmitted gets adjusted by the correspondence framework. AEMAC plan gave a huge Throughput contrasted with the other two plans. Along these lines an increment in the Transmission Rate can be a viable strategy to expand the Throughput in a remote sensor system.

- An increment in Transmission Rate at first gave a slight increment in Delay for SMAC and AEMAC plans. AEMAC plan gave the base Delay alongside ZMAC plan and SMAC plan gave the most extreme Delay. So static plans demonstrate to give higher Delay in a remote sensor system. Likewise, the increment in Transmission Rate has next to no impact on the Delay experienced by the information bundles in a remote sensor system.

- An increment in the Transmission Rate gave a superior Delivery Ratio to AEMAC and ZMAC plans. The Delivery Ratio of SMAC plan which was at first higher than ZMAC plan for a lower Transmission Rate, stooped to a low esteem on an increment in the Transmission Rate. Accordingly, in a remote sensor organize, the better execution of the static plans as far as Delivery Ratio gets corrupted on an increment in the Transmission Rate. Additionally, the increment in the Transmission Rate can give a superior Delivery Ratio in a remote sensor system which utilizes successful course choice arrangements.

- As the hub thickness expands, the Delivery Ratio diminishes for all the three plans AEMAC, ZMAC and SMAC. AEMAC plan displays the most elevated Delivery Ratio contrasted with alternate plans.

- As the quantity of streams expands, Delivery Ratio increments somewhat for AEMAC and ZMAC plans. AEMAC plan demonstrates the greatest Delivery Ratio and ZMAC plan gives the base Delivery Ratio.

- For an increment in the Transmission Rate, AEMAC plan could get a much higher Bandwidth contrasted with the SMAC plan. In remote sensor systems, increment in the Transmission Rate can be selected expanding the Bandwidth in the system. 
- On an increment in the Transmission Rate, Fairness increments significantly for the AEMAC plan contrasted with the SMAC plan. So to accomplish better Fairness in a remote sensor arrange, the increment in Transmission Rate can be chosen as an ideal technique.

- The Bandwidth diminishes in a remote sensor system having a higher Error Rate. In any case, the lessening is lesser in a system which receives channel versatile plans along these lines demonstrating the better execution of the AEMAC plan contrasted with the SMAC plan.

- Fairness diminishes in a remote sensor system having a higher Error Rate and the SMAC plan demonstrates a low Fairness contrasted with the AEMAC plan. In a remote sensor system with fluctuating channel conditions, it is hard to accomplish Fairness. The Load Prediction calculation and the Threshold conformity plan of AEMAC plan represents the expanded Fairness, even in a system with a higher mistake rate.

- In a remote sensor system, on an increment in the Error Rate, the Delay increments significantly for SMAC plan contrasted with the AEMAC plan. In the event that appropriate channel versatile plans are utilized as a part of a remote sensor arrange, the Delay experienced by the information bundles can be significantly decreased, which is demonstrated in the predominant execution displayed by the AEMAC plan.

- On an increment in the Error Rate in a remote sensor arrange, the Throughput diminishes. The AEMAC plan gives a much higher Throughput contrasted with the SMAC plan demonstrating that channel versatile conventions can be utilized viably as a part of a remote sensor system, with differing channel conditions.

- On an increment in the Error rate, the Delivery Ratio diminishes at first and afterward remains verging on consistent for both AEMAC and SMAC plans. AEMAC plan demonstrates a superior Delivery Ratio contrasted with the SMAC plan at high blunder rates, demonstrating that the divert versatile methods embraced in the AEMAC plan can give a superior execution in a remote sensor system

\section{OUTLINE ISSUES}

Scope issue is about to have every area in the physical space of enthusiasm inside of the detecting scope of no less than one sensor. There are a few variables or issues that ought to be considered when outlining a system for scope. Contingent upon the specific application that is being viewed as, the target and the capacities of the sensor hubs that are being utilized, numerous variables ought to be considered, subsequent to these influence the scope execution of WSN. When all is said in done, there are a wide range of configuration issues that influence the scope execution of WSN. While it is unfeasible to cover all the conceivable elements, some of them have been examined in this paper and are as per the following:

\subsection{Deployment: Deterministic Vs Arbitrary}

Sensor hubs are sent in a region by either putting them in foreordained areas or having the hubs haphazardly found. Deterministic organization is appropriate just to a little measured sensor system in an available scope. In remote or unfriendly zones, or for military and debacle applications, or for vast measured systems, the careful positions and number of sensor hubs can't be foreordained. Subsequently, arbitrary organization is utilized as a part of such cases. Dropping sensors from a plane would be a case of irregular situation. It is less demanding to build up a scope plan for deterministic arrangement of sensor hubs than for irregular situation. On the other hand, in numerous organizations, it is either illogical or difficult to convey sensor hubs deterministically. (Fan \& Jin, 2010) Cases of deterministic and arbitrary situation are appeared in fig. 1a) and b).

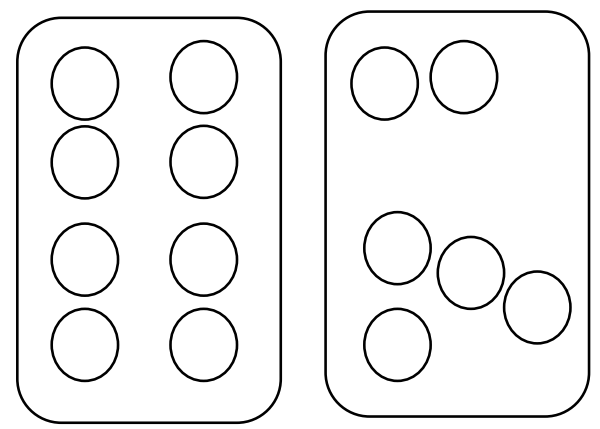

Fig.1 a) Deterministic and b) Random Placement

\subsection{Centralized/Distributed Algorithms}

Once a sensor is allotted a particular work. Then, an algorithm works on it to determine whether there is sufficient coverage in that particular area or not. This leads to the formation of centralized or distributed algorithm. A centralized sensor is the one which consists of a single node or more than it. The location of the nodes is usually near the data sink. Whereas, the distributed algorithm is executed on many or all of nodes (who has gathered information) throughout the network. Thus, the distributed algorithm works on all nodes or on a large no of nodes. As a result, these algorithms are more complex as compared to the centralized algorithms. Fig. 2 a) and b) show distributed and centralized algorithm. The shaded sensors represent the ones which are running a part or all the algorithms.
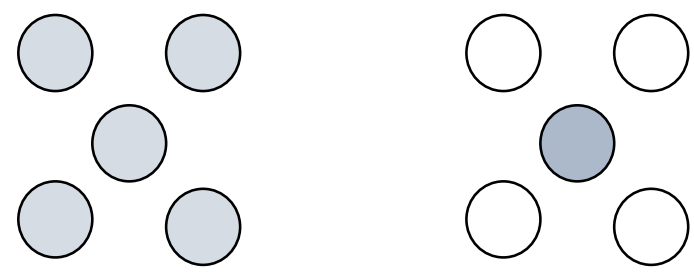

Fig.2 a) Distributed and b) Centralized Algorithm

\subsection{Types of Nodes}

The nodes which are used for a sensor network can be a homogeneous or heterogeneous group of the nodes. The nodes of the homogeneous group have same capabilities. Whereas, in the heterogeneous groups, the power of the nodes is not equally balanced.

\subsection{Sensing Model}

There are two sorts of detecting model - Boolean detecting and probabilistic detecting model. The easiest model is the Boolean model, as per which a hub is equipped for detecting just from focuses that exist in its detecting range and not from any point past it. While, the probabilistic detecting model is a 
more genuine observation, which can be taken as an expansion of the Boolean detecting model, in which the location likelihood of an item diminishes as the separation increments. This model mirrors the conduct of reach detecting gadgets, for example, infrared and ultrasound sensors.

\subsection{Sensing Area}

By and large, the sensors are expected to have the same (or uniform or consistent) detecting extent. For instance, the detecting region is thought to be isotropic (e.g., a round zone in 2-D). A sensor can recognize an article on the off chance that it is in the detecting reach contingent upon its detecting model - Boolean (a sensor can identify an item the length of the item is inside its detecting extent) or probabilistic (the identification likelihood of an article is a component of the separation between the article and the sensor). Likewise, the detecting territory of a sensor hub is extensible to non uniform detecting territories or unpredictable detecting ranges.

\subsection{Types of Coverages}

A significant part of the exploration has been done to create plans incorporating scope and network for WSN. Contingent upon the scope destinations and applications, they can be characterized into three classifications: region scope, point scope, and way coverage (Wang et al., 2003). A brief portrayal is being done here:

Zone scope: Here, the fundamental target of the sensor system is to cover (screen) a zone or a district (the accumulation of all space focuses inside of the sensor field) and to expand the rate of range secured in the given area of interest (ROI).

Point scope: Here, the goal is to cover an arrangement of focuses (focus) with known area that should be checked. The point scope sort centers deciding sensor hub's precise position, in this way, guaranteeing proficient scope application for a predetermined number of stable points(targets).

Way scope: Here, the goal is to minimize the likelihood of undetected infiltration through the obstruction (sensor system) district. It is otherwise called Barrier scope.

\subsection{Coverage Schemes}

\subsubsection{Region (Point Coverage)}

The region scope issue depends on the scope with least sensor hubs and vitality utilization when the district is secured by associated hubs. A locale is said to be k-shrouded, if each point in the given range is secured by atleast $\mathrm{k}$ sensors, where $\mathrm{k}$ is a given parameter.

For each sensor, by ascertaining all convergence focuses between the edges of sensor's detecting zones and between any sensor's detecting region and the limit of this locale, it can assert that the checked district is k-scope by WSN if all crossing point focuses are $\mathrm{k}$-shrouded (Hefeeda \& Bagheri, 2007).This segment audits the different plans utilized as a part of taking care of zone or point scope issue in WSN. The diverse conventions and plans utilized as a part of assessing zone or point scope are depicted as under.

\subsubsection{Scope Configuration Protocol (CCP) (Wang et al., 2003)}

This convention goes for augmenting the quantity of hubs that can be put into rest mode while ensuring k-scope and network. Acc. to (Ghosh \& Das, 2006), scope infers connectivity when the correspondence range $(\mathrm{Rc})$ is more than twice of the detecting range(Rs) i.e. $\mathrm{Rc} \geq 2 \mathrm{Rs}$.
The hubs in CCP can be in any of the three states: SLEEP, LISTEN, and ACTIVE. At first, a hub is in SLEEP state. When it awakens, it enters LISTEN state and in light of the result of the qualification standard over a period interim, it will enter either SLEEP or ACTIVE state.

Every hub will occasionally telecast HELLO message with its own area and status. From this, the hubs will make a table of each of its neighbors when it is in the LISTEN state. On the off chance that its whole detecting territory is secured by its neighbors then it will move into SLEEP mode. It will keep on being in that state until the rest clock lapses and afterward, they will re-assess scope and choose whether to go into the SLEEP state or stay in the ACTIVE state. The CCP convention does not ensure network when Rc $<2$ Rs.

To accomplish this, the arrangement received in (Wang et al., 2003) is to incorporate CCP with SPAN [8] to give both detecting scope and system network. Compass is a conveyed calculation that saves vitality by killing pointless hubs while looking after availability.

The joined qualification principle says: (1) a dormant hub goes into the dynamic state on the off chance that it fulfills the qualification guidelines of SPAN and CCP, and (2) a dynamic hub will pull back in the event that it can't meet the qualification standard of SPAN or CCP

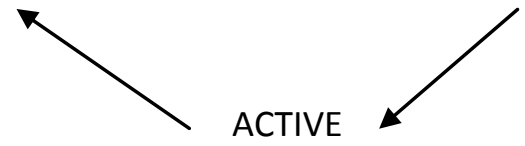

Fig. State Transition in CCP

\subsubsection{Adaptive Self-Configuring Sensor Networks Topologies (ASCENT)}

Rising depends on the idea that, as thickness increments, just a subset of the hubs is important to build up a steering sending spine. The primary thought of ASCENT is to let sensors survey their availability and in addition their information misfortune rate and enact their neighbors in view of these nearby estimations.

Acc. to (Chi-Fu \& Yu-Chee, 2005), ASCENT comprises of a few stages. At the point when a hub first introduces, it goes into a listening-just stage called neighbor revelation stage, where every hub acquires an assessment of the quantity of neighbors effectively transmitting messages in view of nearby estimations.

After this, hubs go into the join choice stage, where they choose whether to join the multi-bounce dissemination sensor system and a hub might briefly join the system for a sure timeframe to test whether it adds to enhanced availability.

On the off chance that it joins the system for a more extended time, it goes into a dynamic stage and begins sending directing control and information messages.

On the off chance that it doesn't, then it goes into the versatile stage, where it turns itself off for a timeframe, or decreases its transmission range. The downside of ASCENT is that working hubs never do a reversal to rest. 


\subsubsection{Optimal Geographical Density Control $(O G D C)$}

This is comparative in numerous regards to CCP yet contrasts in that it tries to minimize the covering of detecting ranges of all sensor hubs for situations when Rc $>2$ Rs. The calculation begins with every one of the hubs at first in "UNDECIDED" state. Whenever, a hub can be in one of the tree states: UNDECIDED, ON, and OFF.

In the event that adequate force is accessible, and the region is not completely secured, then the hub includes the beginning hub as the neighbor, sets its state to "ON" and shows the force on message once more. This procedure proceeds with somewhat diverse conduct for the force on messages got from beginning and non-beginning hubs.

This is a decentralized and confined thickness control plan in view of the evidence that if $\mathrm{Rc}>2 \mathrm{Rs}$, then a scope suggests network. The objective of OGDC is to augment the quantity of resting sensors while guaranteeing scope and availability.

By utilizing its own area and the working sensor's area, a sensor can check regardless of whether it turns on. In OGDC, sensor hubs need precise area data and time synchronization and working hubs never do a reversal to rest yet distinctive hubs may be working in diverse adjusts so vitality utilization may in any case be adjusted among every one of the hubs. (Hefeeda \& Bagheri, 2007)

\subsubsection{Lightweight Arrangement Mindful Planning (LDAS)}

The goal of LDAS is to expand system lifetime and additionally adequate detecting territories. This strategy examines the excess detecting zones among neighboring remote sensors. It is accepted that sensor hubs are not outfitted with GPS or different gadgets to get area data, thus, LDAS gives tight upper and lower limits on the likelihood of complete repetition and on the normal halfway excess.

In LDAS, when the quantity of working neighbors surpasses an edge controlled by the application's prerequisite on detecting scope, the hub haphazardly chooses some of its neighbors to kill and issues tickets to them. At the point when a hub gathers enough tickets from its neighbors, it may enter the enjoying some downtime mode after an arbitrary back-off period. (Zhou et al., 2004)

\subsubsection{For Barrier or Path Coverage}

All plans talked about above have expected that the WSN is sent for checking (detecting) occasions happening in sending locale (e.g., all area or some static focuses) and in this way, the idea of infiltration scope in the scope plan configuration has been overlooked.

In fact, numerous applications may require considering about the objective entering through the scope district by WSN. For instance, sensor system sending in a combat zone is to identify foe developments.

It has been appeared (Fan \& Jin, 2010) in the maximal rupture (bolster) way are characterized as the way on which the separation from any point to the nearest sensor is boosted (minimized).

By consolidating computational geometry and chart theoretic systems, particularly the Voronoi outline, the Delaunay triangulation, and diagram seek calculations, polynomial time calculations are proposed to discover such ways.

Other than from the break furthermore, bolster ways, the idea of time ought to be incorporated to mirror the more reasonable likelihood of a moving target being detected subsequent to the detecting capacity of sensors can be enhanced as the distributed detecting time (presentation) increments.

Voronoi graph can be utilized as one of the examining strategies in deciding WSN scope; with the sensors go about as the locales if all Voronoi polygons vertices are secured then the ROI is completely secured generally scope openings exist. Delaunay triangle is framed by three locales gave if and just if the destinations circumcircle does not contain different locales.

The middle purpose of the circle is a Voronoi vertex with equivalent separation from each of the three locales. The work demonstrated that a maximal break way must lie on the edges of Voronoi chart while maximal introduction way lie on the edges of Delaunay triangulation.

\section{COMPARATIVE ANALYSIS}

In the wake of concentrating on the different plans of area(point) scope, we now do the similar investigation of the distinctive plans concentrated as such.

This investigation is done as per the examination given in (Fan \& Jin, 2010), utilizing the parameters, for example, goal of the particular plans, their sending technique, algorithmic trademark utilized, detecting model to judge the detecting range and detecting region and their separate purpose of disappointment which goes about as a confinement of the plans. The table given beneath demonstrates the correlation of distinctive plans.

Table 1: Comparison of different coverage schemes for area coverage of WSN

\begin{tabular}{|l|l|l|l|l|l|l|}
\hline \multirow{2}{*}{ Schemes } & \multicolumn{5}{|c|}{ Features } \\
\cline { 2 - 7 } & Objective & $\begin{array}{l}\text { Deployment } \\
\text { method }\end{array}$ & Sensing model & $\begin{array}{l}\text { Algorithmic } \\
\text { Characteristic }\end{array}$ & Sensing area & Point of failure \\
\hline CCP & $\begin{array}{l}\text { Maximize } \\
\text { number of } \\
\text { sleeping nodes }\end{array}$ & $\begin{array}{l}\text { Random or } \\
\text { Deterministic }\end{array}$ & Boolean & Distributed & Circular (2-D) & $\begin{array}{l}\text { No network } \\
\text { connectivity if } \\
\text { Rc }<2 R s\end{array}$ \\
\hline ASCENT & $\begin{array}{l}\text { Assess } \\
\text { connectivity } \\
\text { and data loss } \\
\text { rate }\end{array}$ & $\begin{array}{l}\text { Random or } \\
\text { Deterministic }\end{array}$ & Probabilistic & Centralized & Any & $\begin{array}{l}\text { Working nodes } \\
\text { never go back } \\
\text { to sleep }\end{array}$ \\
\hline OGDC & $\begin{array}{l}\text { Minimize the } \\
\text { overlapping } \\
\text { area of nodes }\end{array}$ & $\begin{array}{l}\text { Random or } \\
\text { Deterministic }\end{array}$ & Boolean & Distributed & Circular (2-D) & $\begin{array}{l}\text { Working nodes } \\
\text { never go back } \\
\text { to sleep }\end{array}$ \\
\hline LDAS & $\begin{array}{l}\text { Maximize } \\
\text { network } \\
\text { lifetime and } \\
\text { sensing area }\end{array}$ & $\begin{array}{l}\text { Random or } \\
\text { Deterministic }\end{array}$ & Boolean & Distributed & Circular (2-D) & $\begin{array}{l}\text { Redundant } \\
\text { sensing areas }\end{array}$ \\
\hline
\end{tabular}




\section{CONCLUSION}

This paper covered and analyzed the various coverage problems occurring in the WSN and various changes in the design that should be made for its better performance were considered. It also included the types of coverage, namely, area coverage, point coverage and barrier coverage. The present research works on calculating and improving the coverage performance of a point and path coverage. This paper focused on various coverage schemes and their comparative analysis. These schemes were analyzed and compared on different parameters such as deployment strategy, objective, algorithms used, features, sensing range and sensing model and point of failures. Overall, this involves many problems on which work needs to be done. By doing so, these wireless sensor can give the best performance.

\section{REFERENCES}

[1] Akyildiz, I. F., Su, W., Sankarasubramaniam, Y., \& Cayirci, E. (2002). Wireless sensor networks: a survey. Computer networks, 38(4), 393-422.

[2] Chi-Fu, H., \& Yu-Chee, T. (2005). The Coverage Problem in a Wireless Sensor Network. Mobile Networks and Applications, 10(4), 519.

[3] Fan, G., \& Jin, S. (2010). Coverage problem in wireless sensor network: A survey. Journal of networks, 5(9), 1033-1040

[4] Ghosh, A., \& Das, S. K. (2006). Coverage and connectivity issues in wireless sensor networks. Mobile, wireless, and sensor networks: Technology, applications, and future directions, 221-256.

[5] Hefeeda, M., \& Bagheri, M. (2007). Randomized kcoverage algorithms for dense sensor networks. Paper presented at the INFOCOM 2007. 26th IEEE International Conference on Computer Communications. IEEE.

[6] Polastre, J., Szewczyk, R., Mainwaring, A., Culler, D., \& Anderson, J. (2004). Analysis of wireless sensor networks for habitat monitoring Wireless sensor networks (pp. 399-423): Springer.

[7] Wang, X., Xing, G., Zhang, Y., Lu, C., Pless, R., \& Gill, C. (2003). Integrated coverage and connectivity configuration in wireless sensor networks. Paper presented at the Proceedings of the 1st international conference on Embedded networked sensor systems.

[8] Zhou, Z., Das, S., \& Gupta, H. (2004). Connected kcoverage problem in sensor networks. Paper presented at the Computer Communications and Networks, 2004. ICCCN 2004. Proceedings. 13th International Conference on.

[9] I.F. Akyildiz, W. Su, A power aware enhanced routing (PAER) protocol for sensor networks, Georgia Tech Technical Report, January 2002

[10] A. Bakre, B.R. Badrinath, I-TCP: indirect TCP for mobile hosts, Proceedings of the 15th International Conference on Distributed Computing Systems, Vancouver, BC, May 1995, pp. 136-143

[11] M. Bhardwaj, T. Garnett, A.P. Chandrakasan, Upper bounds on the lifetime of sensor networks, IEEE International Conference on Communications ICC'01, Helsinki, Finland, June 2001

[12] V. R. Vatsa, G. Singh (2015). A Literature review of Internet of Things (IOT), International Journal of Computer Systems, Volume 2, Issue 8, August, 2015,355-358.

[13] B.G. Celler et al., An instrumentation system for the remote monitoring of changes in functional health status of the elderly, International Conference IEEE-EMBS, New York, 1994, pp. 908-909

[14] A. Cerpa, D. Estrin, ASCENT: adaptive self-configuring sensor networks topologies, UCLA Computer Science Department Technical Report UCLA/CSDTR-01-0009, May 2001

[15] A. Cerpa, J. Elson, M. Hamilton, J. Zhao, Habitat monitoring: application driver for wireless communications technology, ACM SIGCOMM'2000, Costa Rica, April 2001

[16] J.M. Cramer, R.A. Scholtz, M.Z. Win, On the analysis of UWB communication channels, IEEE MILCOM'99, 1999, pp. 1191-1195

[17] D. Estrin, R. Govindan, J. Heidemann, S. Kumar, Next century challenges: scalable coordination in sensor networks, ACM MobiCom'99, Washington, USA, 1999, pp. $263-270$ 